\title{
Classical access structures of ramp secret sharing based on quantum stabilizer codes
}

\author{
Ryutaroh Matsumoto ${ }^{1,2}$ (D)
}

Received: 19 April 2019 / Accepted: 13 November 2019 / Published online: 26 November 2019

(c) The Author(s) 2019

\begin{abstract}
In this paper, we consider to use the quantum stabilizer codes as secret sharing schemes for classical secrets. We give necessary and sufficient conditions for qualified and forbidden sets in terms of quantum stabilizers. Then, we give a Gilbert-Varshamovtype sufficient condition for existence of secret sharing schemes with given parameters, and by using that sufficient condition, we show that roughly $19 \%$ of participants can be made forbidden independently of the size of classical secret, in particular when an $n$-bit classical secret is shared among $n$ participants having 1 -qubit share each. We also consider how much information is obtained by an intermediate set and express that amount of information in terms of quantum stabilizers. All the results are stated in terms of linear spaces over finite fields associated with the quantum stabilizers.
\end{abstract}

Keywords Secret sharing · Quantum error-correcting code · Gilbert-Varshamov bound

Mathematics Subject Classification 94A62 $\cdot 81 \mathrm{P} 70 \cdot 94 \mathrm{~B} 65$

\section{Introduction}

Secret sharing is a scheme to share a secret among multiple participants so that only qualified sets of participants can reconstruct the secret, while forbidden sets have no

\footnotetext{
An extended abstract [28] of this manuscript appeared in https://www.lebesgue.fr/content/sem2019WCC-programthe Proceedings of the Eleventh International Workshop on Coding and Cryptography (WCC 2019), Saint-Jacut-de-la-Mer, France, March 31-April 5, 2019. Its presentation slides are available from https://www.slideshare.net/RyutarohMatsumoto. This research is partly supported by JSPS Grant No. 17K06419.

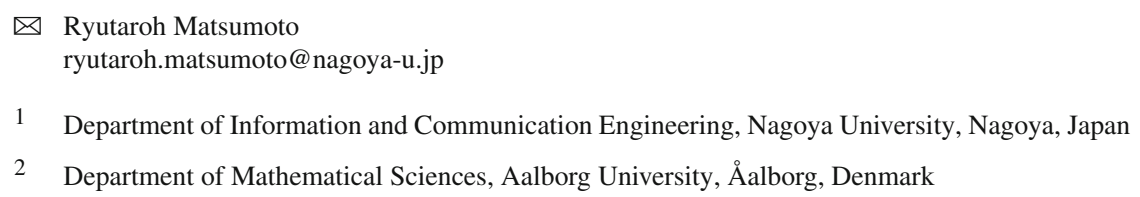


information about the secret [36]. A piece of information received by a participant is called a share. A set of participants that is neither qualified nor forbidden is said to be intermediate. Both secret and shares are traditionally classical information. There exists a close connection between secret sharing and classical error-correcting codes $[3,7,10,11,19,23,31]$.

After the importance of quantum information became well-recognized, secret sharing schemes with quantum shares were proposed [8,15-17,37]. A connection between quantum secret sharing and quantum error-correcting codes has been well known for many years $[8,13,15,21,22,35,37]$, none of which has determined the access structure of secret sharing schemes with classical secrets and quantum shares constructed from quantum stabilizer codes. The well-known classes of quantum error-correcting codes are the CSS codes $[6,38]$, the stabilizer codes $[4,5,14]$ that include the CSS codes as a special case, and their non-binary generalizations [2,18,29].

The access structure of a secret sharing scheme is the set of qualified sets, that of intermediate sets and that of forbidden sets. For practical use of secret sharing, one needs sufficient (and desirably necessary) conditions on qualified sets and forbidden sets. It is natural to investigate access structures of secret sharing schemes constructed from quantum error-correcting codes. For secret sharing schemes with quantum secret and quantum shares, necessary and sufficient conditions for qualified sets and forbidden sets were clarified for the CSS codes [26,37] and the stabilizer codes [13,25]. For classical secret and quantum shares, the access structure was clarified in [26, Section 4.1] with [33, Theorem 1] for the CSS codes but has not been clarified for secret sharing schemes based on quantum stabilizer codes, as far as this author knows.

Advantages of using quantum shares for sharing a classical secret are that we can have smaller size of shares [15, Section 4] and that we can realize access structures that cannot be realized by classical shares [24,27]. For example, it is well known that the size of classical shares cannot be smaller than that of the classical secret in a perfect secret sharing scheme, where perfect means that there is no intermediate set, while ramp or non-perfect means that there exist intermediate sets [39]. On the other hand, the superdense coding can be a secret sharing scheme sharing 2 bits by 2 qubits sent to 2 participants [15, Section 4]. Any participant has no information about the secret, while the 2 participants can reconstruct the secret. We see a perfect threshold scheme sharing 2-bit classical secret by 1-qubit shares. This paper will generalize Gottesman's secret sharing [15, Section 4] to the arbitrary number of participants and the arbitrary size of classical secrets.

In this paper, we give necessary and sufficient conditions for qualified and forbidden sets in terms of the underlying linear spaces over finite fields of quantum stabilizers in Sect. 3, after introducing necessary notations in Sect. 2. Section 3 also includes sufficient conditions in terms of a quantity similar to relative generalized Hamming weight [20] of classical linear codes related to the quantum stabilizers. We also consider how much information is obtained by an intermediate set and express that amount of information in terms of the underlying linear spaces of quantum stabilizers in Sect. 4. Then, we translate our theorems over prime finite fields by the symplectic inner product into arbitrary finite fields, the Euclidean, and the hermitian inner products in Sect. 5. Section 5 also includes an elementary construction by the Reed-Solomon codes as an example of Sect. 5.3. Finally, we give a Gilbert-Varshamov-type sufficient condition 
for existence of secret sharing schemes with given parameters, and by using that sufficient condition, we show that roughly $19 \%$ of participants can be made forbidden independently of the size of classical secret, which cannot be realized by classical shares, in Sect. 6. Concluding remarks are given in Sect. 7. The extended abstract [28] in the workshop had no mathematical proofs and only few examples due to space limitation, and there were confusing typographical errors in the main theorems [28, Theorems 18 and 19].

\section{Notations}

Let $p$ be a prime number, $\mathbf{F}_{p}$ the finite field with $p$ elements, and $\mathbf{C}_{p}$ the $p$-dimensional complex linear space. The quantum state space of $n$ qudits is denoted by $\mathbf{C}_{p}^{\otimes n}$ with its orthonormal basis $\left\{|\mathbf{v}\rangle: \mathbf{v} \in \mathbf{F}_{p}^{n}\right\}$.

For two vectors $\mathbf{a}, \mathbf{b} \in \mathbf{F}_{p}^{n}$, denote by $\langle\mathbf{a}, \mathbf{b}\rangle_{E}$ the standard Euclidean inner product. For two vectors $(\mathbf{a} \mid \mathbf{b})$ and $\left(\mathbf{a}^{\prime} \mid \mathbf{b}^{\prime}\right) \in \mathbf{F}_{p}^{2 n}$, we define the standard symplectic inner product

$$
\left\langle(\mathbf{a} \mid \mathbf{b}),\left(\mathbf{a}^{\prime} \mid \mathbf{b}^{\prime}\right)\right\rangle_{S}=\left\langle\mathbf{a}, \mathbf{b}^{\prime}\right\rangle_{E}-\left\langle\mathbf{a}^{\prime}, \mathbf{b}\right\rangle_{E}
$$

For an $\mathbf{F}_{p}$-linear space $C \subset \mathbf{F}_{p}^{2 n}, C^{\perp s}$ denotes its orthogonal space in $\mathbf{F}_{p}^{2 n}$ with respect to $\langle\cdot, \cdot\rangle_{s}$. Throughout this paper, we always assume $\operatorname{dim} C=n-k$ and $C \subseteq C^{\perp s}$.

For $(\mathbf{a} \mid \mathbf{b}) \in \mathbf{F}_{p}^{2 n}$, define the $p^{n} \times p^{n}$ complex unitary matrix $X(\mathbf{a}) Z(\mathbf{b})$ as defined in [18]. An $[[n, k]]_{p}$ quantum stabilizer codes $Q$ encoding $k$ qudits into $n$ qudits can be defined as a simultaneous eigenspace of all $X(\mathbf{a}) Z(\mathbf{b})((\mathbf{a} \mid \mathbf{b}) \in C)$. Unlike [18], we do not require the eigenvalue of $Q$ to be one.

It is well known in mathematics [1, Chapter 7] that there always exists $C \subseteq C_{\max } \subseteq$ $C^{\perp s}$ such that $C_{\max }=C_{\max }^{\perp s}$. Note that $C_{\max }$ is not unique and usually there are many possible choices of $C_{\max }$. We have $\operatorname{dim} C_{\max }=n$ and have an isomorphism $f: \mathbf{F}_{p}^{k} \rightarrow C^{\perp s} / C_{\max }$ as linear spaces without inner products. Since $C_{\max }=C_{\max }^{\perp s}$, $C_{\max }$ defines an $[[n, 0]]_{p}$ quantum stabilizer code $Q_{0}$. Without loss of generality, we may assume $Q_{0} \subset Q$. Let $|\varphi\rangle \in Q_{0}$ be a quantum state vector. Since $C_{\max }=C_{\max }^{\perp s}$, for a coset $V \in C^{\perp s} / C_{\max }$ and $(\mathbf{a} \mid \mathbf{b}),\left(\mathbf{a}^{\prime} \mid \mathbf{b}^{\prime}\right) \in V, X(\mathbf{a}) Z(\mathbf{b})|\varphi\rangle$ and $X\left(\mathbf{a}^{\prime}\right) Z\left(\mathbf{b}^{\prime}\right)|\varphi\rangle$ differ by a constant multiple in $\mathbf{C}$ and physically express the same quantum state in $Q$. By an abuse of notation, for a coset $V \in C^{\perp s} / C_{\max }$ we will write $|V \varphi\rangle$ to mean $X(\mathbf{a}) Z(\mathbf{b})|\varphi\rangle((\mathbf{a} \mid \mathbf{b}) \in V)$.

For a given classical secret $\mathbf{m} \in \mathbf{F}_{p}^{k}$, we consider the following secret sharing scheme with $n$ participants:

1. $f(\mathbf{m})$ is a coset of $C^{\perp s} / C_{\max }$. Prepare the quantum codeword $|f(\mathbf{m}) \varphi\rangle \in Q$ that corresponds to the classical secret $\mathbf{m}$.

2. Distribute each qudit in the quantum codeword $|f(\mathbf{m}) \varphi\rangle$ to a participant.

We can also consider a secret sharing scheme for a $k$-qudit secret $|\mathbf{m}\rangle$ with $n$ participants as follows. The reason why we also consider secret sharing schemes with quantum secrets is to contrast the difference between the classical and the quantum 
access structures of a secret sharing scheme constructed from the same quantum stabilizer in Remarks 7 and 10, while the main focus of the present paper is to share classical secrets.

1. Encode a given quantum secret $\sum_{\mathbf{m} \in \mathbf{F}_{p}^{k}} \alpha(\mathbf{m})|\mathbf{m}\rangle$ into the quantum codeword $\sum_{\mathbf{m} \in \mathbf{F}_{p}^{k}} \alpha(\mathbf{m})|f(\mathbf{m}) \varphi\rangle \in Q$, where $\alpha(\mathbf{m}) \in \mathbf{C}$ are complex coefficients with $\sum_{\mathbf{m} \in \mathbf{F}_{p}^{k}}|\alpha(\mathbf{m})|^{2}=1$.

2. Distribute each qudit in the quantum codeword $\sum_{\mathbf{m} \in \mathbf{F}_{p}^{k}} \alpha(\mathbf{m})|f(\mathbf{m}) \varphi\rangle$ to a participant.

Let $A \subset\{1, \ldots, n\}$ be a set of shares (or equivalently participants), $\bar{A}=$ $\{1, \ldots, n\} \backslash A$, and $\operatorname{Tr}_{\bar{A}}$ the partial trace over $\bar{A}$. For a density matrix $\rho, \operatorname{col}(\rho)$ denotes its column space. When $\operatorname{col}\left(\rho_{1}\right), \ldots, \operatorname{col}\left(\rho_{n}\right)$ are orthogonal to each other, that is, $\rho_{i} \rho_{j}=0$ for $i \neq j$, we can distinguish $\rho_{1}, \ldots, \rho_{n}$ by a suitable projective measurement with probability 1 .

Definition 1 We say $A$ to be $c$-qualified (classically qualified) if $\operatorname{col}\left(\operatorname{Tr}_{\bar{A}}(|f(\mathbf{m}) \varphi\rangle\right.$ $\langle f(\mathbf{m}) \varphi|))$ and $\operatorname{col}\left(\operatorname{Tr}_{\bar{A}}\left(\left|f\left(\mathbf{m}^{\prime}\right) \varphi\right\rangle\left\langle f\left(\mathbf{m}^{\prime}\right) \varphi\right|\right)\right)$ are orthogonal to each other for different $\mathbf{m}, \mathbf{m}^{\prime} \in \mathbf{F}_{p}^{k}$. We say $A$ to be $c$-forbidden (classically forbidden) if $\operatorname{Tr}_{\bar{A}}(|f(\mathbf{m}) \varphi\rangle\langle f(\mathbf{m}) \varphi|)$ is the same density matrix regardless of classical secret $\mathbf{m}$. By a classical access structure, we mean the set of $c$-qualified sets and the set of $c$-forbidden sets.

For a quantum secret, the quantum qualified ( $q$-qualified) sets and the quantum forbidden ( $q$-forbidden) sets are mathematically defined in [33]. By a quantum access structure, we mean the set of $q$-qualified sets and the set of $q$-forbidden sets.

Remark 2 When classical shares on $A$ are denoted by $S_{A}$, the conventional definition of qualifiedness is $I\left(\mathbf{m} ; S_{A}\right)=H(\mathbf{m})$ and that of forbiddenness is $I\left(\mathbf{m} ; S_{A}\right)=0$ [39], where $H(\cdot)$ denotes the entropy and $I(\cdot ; \cdot)$ denotes the mutual information [9]. Let $\rho_{A}=\sum_{\mathbf{m} \in \mathbf{F}_{p}^{k}} p(\mathbf{m}) \operatorname{Tr}_{\bar{A}}(|f(\mathbf{m}) \varphi\rangle\langle f(\mathbf{m}) \varphi|)$, where $p(\mathbf{m})$ is the probability distribution of classical secrets $\mathbf{m}$. The quantum counterpart of mutual information for classical random variables is the Holevo information $I\left(\mathbf{m} ; \rho_{A}\right)$ [32, Section 12.1.1]. $A$ is $c$-qualified if and only if $I\left(\mathbf{m} ; \rho_{A}\right)=H(\mathbf{m})$, and is $c$-forbidden if and only if $I\left(\mathbf{m} ; \rho_{A}\right)=0$. Therefore, Definition 1 is a natural generalization of the conventional definition in [39].

Example 3 We will see how one can express the secret sharing scheme based on superdense coding [15, Section 4] by a quantum stabilizer. Let $p=2, n=2$ and $C$ be the zero-dimensional linear space consisting of only the zero vector. Then, $C^{\perp s}=\mathbf{F}_{2}^{4}$. We choose $C_{\max }$ as the space spanned by $(1,1 \mid 0,0)$ and $(0,0 \mid 1,1)$. For a classical secret $\left(m_{1}, m_{2}\right) \in \mathbf{F}_{2}^{2}$, define the map $f$ as $f\left(m_{1}, m_{2}\right)=\left(m_{1}, 0 \mid m_{2}, 0\right)+C_{\max } \in C^{\perp s} / C_{\max }$. We can choose $[[2,0]]_{2}$ quantum code $Q_{0}$ as the one-dimensional complex linear space spanned by the Bell state

$$
|\varphi\rangle=\frac{|00\rangle+|11\rangle}{\sqrt{2}},
$$


which corresponds to the two-bit secret $(0,0)$. The secret $\left(m_{1}, m_{2}\right)$ is encoded to

$$
X\left(m_{1}, 0\right) Z\left(m_{2}, 0\right)|\varphi\rangle=\frac{\left|m_{1} 0\right\rangle+(-1)^{m_{2}}\left|\left(1-m_{1}\right) 1\right\rangle}{\sqrt{2}} .
$$

It is clear that the share set $\{1,2\}$ is $c$-qualified. When $A=\{1\}$ or $A=\{2\}$, we have

$$
\operatorname{Tr}_{\bar{A}}(|f(\mathbf{m}) \varphi\rangle\langle f(\mathbf{m}) \varphi|)=\frac{1}{2}\left(\begin{array}{ll}
1 & 0 \\
0 & 1
\end{array}\right) \text {, }
$$

which means $\{1\},\{2\}$ and $\varnothing$ are $c$-forbidden. We have determined the classical access structure completely, and we see that this scheme is perfect [39] in the sense that there is no intermediate set.

For completeness, we also note its quantum access structure. The set $\{1,2\}$ is $q$ qualified and $\emptyset$ is $q$-forbidden, of course. By [25, Eq. (3)], we see that $\{1\}$ and $\{2\}$ are intermediate, that is, neither qualified nor forbidden. This quantum access structure exemplifies the fact that $q$-qualifiedness implies $c$-qualifiedness, that $q$-forbiddenness implies $c$-forbiddenness and that their converses are generally false [33, Theorems 1 and 2]. It also exemplifies the fact that if quantum secret is larger than quantum shares, then the scheme cannot be perfect $[8,15]$.

\section{Necessary and sufficient conditions on classically qualified and classically forbidden sets}

Let $A \subset\{1, \ldots, n\}$. Define $\mathbf{F}_{p}^{A}=\left\{\left(a_{1}, \ldots, a_{n} \mid b_{1}, \ldots, b_{n}\right) \in \mathbf{F}_{p}^{2 n}:\left(a_{i}, b_{i}\right)=0\right.$ for $i \notin A\}$. Let $P_{A}$ be the projection map onto $A$, that is, $P_{A}\left(a_{1}, \ldots, a_{n} \mid b_{1}, \ldots, b_{n}\right)=$ $\left(a_{i} \mid b_{i}\right)_{i \in A}$.

Theorem 4 For the secret sharing scheme described in Sect. 2, A is c-qualified if and only if

$$
\operatorname{dim} C_{\max } / C=\operatorname{dim} C_{\max } \cap \mathbf{F}_{p}^{A} / C \cap \mathbf{F}_{p}^{A} .
$$

A is c-forbidden if and only if

$$
0=\operatorname{dim} C_{\max } \cap \mathbf{F}_{p}^{A} / C \cap \mathbf{F}_{p}^{A}
$$

The proof is given after showing two examples below.

Example 5 Consider the situation in Example 3. For $A=\{1\}$ or $A=\{2\}$, we see that $C_{\max } \cap \mathbf{F}_{2}^{A}$ and $C \cap \mathbf{F}_{2}^{A}$ are the zero linear space and that Eq. (2) holds. For $A=\{1,2\}$, Eq. (1) is clearly true.

Example 6 In this example, we show that a different choice of $C_{\max }$ gives a different access structure. Let $C$ be as Example 5 and $C_{\max }$ be the linear space generated by $(0,0 \mid 1,0)$ and $(0,0 \mid 0,1)$. A classical secret $\left(m_{1}, m_{2}\right)$ is now encoded to $\left|m_{1} m_{2}\right\rangle$. For $A=\{1\}$ or $A=\{2\}$, both (1) and (2) are false and both $A=\{1\}$ and $A=\{2\}$ are intermediate sets. This example shows that the choice of $C_{\max }$ is important. 
Proof (Theorem 4) Assume Eq. (1). Then, there exists a basis $\left\{\left(\mathbf{a}_{1} \mid \mathbf{b}_{1}\right)+C, \ldots,\left(\mathbf{a}_{k} \mid \mathbf{b}_{k}\right)\right.$ $+C\}$ of $C_{\max } / C$ such that $\left(\mathbf{a}_{i} \mid \mathbf{b}_{i}\right) \in \mathbf{F}_{p}^{A}$. Since $C_{\max }^{\perp s}=C_{\max }$, any two vectors in a coset $V \in C^{\perp} / C_{\max }$ have the same value of the symplectic inner product against a fixed $\left(\mathbf{a}_{i} \mid \mathbf{b}_{i}\right)$, which will be denoted by $\left\langle\left(\mathbf{a}_{i} \mid \mathbf{b}_{i}\right), V\right\rangle_{s}$. Suppose that we have two different cosets $V_{1}, V_{2} \in C^{\perp} / C_{\max }$, and that $\left\langle\left(\mathbf{a}_{i} \mid \mathbf{b}_{i}\right), V_{1}\right\rangle_{s}=\left\langle\left(\mathbf{a}_{i} \mid \mathbf{b}_{i}\right), V_{2}\right\rangle_{s}$ for all $i$. Since $C_{\max }^{\perp s}=C_{\max }$, it means that $V_{1}-V_{2}=C_{\max }$ is zero in $C^{\perp} / C_{\max }$, a contradiction. We have seen that any two different cosets have different symplectic inner product values against some $\left(\mathbf{a}_{i} \mid \mathbf{b}_{i}\right)$. For each $i$, the $n$ participants can collectively perform quantum projective measurement corresponding to the eigenspaces of $X\left(\mathbf{a}_{i}\right) Z\left(\mathbf{b}_{i}\right)$ and can determine the symplectic inner $\operatorname{product}^{1}\left\langle\left(\mathbf{a}_{i} \mid \mathbf{b}_{i}\right), f(\mathbf{m})\right\rangle_{s}$ as [18, Lemma 5] when the classical secret is $\mathbf{m}$. Since $\left(\mathbf{a}_{i} \mid \mathbf{b}_{i}\right)$ has nonzero components only at $A$, the above measurement can be done only by $A$, which means $A$ can reconstruct $\mathbf{m}$.

Assume that Eq. (1) is false. Since the orthogonal space of $C$ in $\mathbf{F}_{p}^{A}$ is isomorphic to $P_{A}\left(C^{\perp s}\right)$, which can be seen as the almost same argument as the duality between shortened linear codes and punctured linear codes [34], we see that $\operatorname{dim} P_{A}\left(C^{\perp s}\right) / P_{A}\left(C_{\max }\right)<\operatorname{dim} C^{\perp s} / C_{\max }$. This means that there exists two different classical secrets $\mathbf{m}_{1}$ and $\mathbf{m}_{2}$ such that $P_{A}\left(f\left(\mathbf{m}_{1}\right)\right)=P_{A}\left(f\left(\mathbf{m}_{2}\right)\right)$. This means that the encoding procedures of $\mathbf{m}_{1}$ and $\mathbf{m}_{2}$ are exactly same on $A$ and produce the same density matrix on $A$, which shows that $A$ is not $c$-qualified.

Assume Eq. (2). Then, we have $\operatorname{dim} P_{A}\left(C^{\perp s}\right) / P_{A}\left(C_{\max }\right)=0$. This means that for all classical secrets $\mathbf{m}, P_{A}(f(\mathbf{m}))$ and their encoding procedures on $A$ are same, which produces the same density matrix on $A$ regardless of $\mathbf{m}$. This shows that $A$ is $c$-forbidden.

Assume that Eq. (2) is false. Then, there exist two different classical secrets $\mathbf{m}_{1}$, $\mathbf{m}_{2}$, and $(\mathbf{a} \mid \mathbf{b}) \in C_{\max } \cap \mathbf{F}_{p}^{A} \backslash C \cap \mathbf{F}_{p}^{A}$ such that

$$
\left\langle(\mathbf{a} \mid \mathbf{b}), f\left(\mathbf{m}_{1}\right)\right\rangle_{s} \neq\left\langle(\mathbf{a} \mid \mathbf{b}), f\left(\mathbf{m}_{2}\right)\right\rangle_{s} .
$$

By [18, Lemma 5], this means that the quantum measurement corresponding to $X(\mathbf{a}) Z(\mathbf{b})$ gives different outcomes with $\operatorname{Tr}_{\bar{A}}\left(\left|f\left(\mathbf{m}_{1}\right) \varphi\right\rangle\left\langle f\left(\mathbf{m}_{\mathbf{1}}\right) \varphi\right|\right)$ and $\operatorname{Tr}_{\bar{A}}$ $\left(\left|f\left(\mathbf{m}_{2}\right) \varphi\right\rangle\left\langle f\left(\mathbf{m}_{2}\right) \varphi\right|\right)$. Since $(\mathbf{a} \mid \mathbf{b}) \in \mathbf{F}_{p}^{A}$, measurement of $X(\mathbf{a}) Z(\mathbf{b})$ can be performed only by participants in $A$. These observations show that $A$ is not $c$-forbidden.

Remark 7 A necessary and sufficient condition for $A$ being $q$-qualified is [25, Eq. (3)]

$$
C^{\perp s} \cap \mathbf{F}_{p}^{\bar{A}}=C_{\max } \cap \mathbf{F}_{p}^{\bar{A}}=C \cap \mathbf{F}_{p}^{\bar{A}}
$$

Since $\operatorname{ker}\left(P_{A}\right)=\mathbf{F}_{p}^{\bar{A}}$, we have $\operatorname{dim} P_{A}\left(C^{\perp s}\right) / P_{A}\left(C_{\max }\right)=k$. The relation between duals of punctured codes and shortened codes [34] implies $\operatorname{dim} C_{\max } \cap \mathbf{F}_{p}^{A} / C \cap \mathbf{F}_{p}^{A}=k$. Therefore, Eq. (3) implies Eq. (1).

\footnotetext{
1 If we assume a non-prime finite field $\mathbf{F}_{q}$ as our base field, then the quantum measurement outcome just determines [18, Lemma 5] $\operatorname{Tr}_{q / p}\left(\left\langle\left(\mathbf{a}_{i} \mid \mathbf{b}_{i}\right), f(\mathbf{m})\right\rangle_{s}\right)$ in place of $\left\langle\left(\mathbf{a}_{i} \mid \mathbf{b}_{i}\right), f(\mathbf{m})\right\rangle_{s}$, where $\operatorname{Tr}_{q / p}$ is the trace map from $\mathbf{F}_{q}$ to its prime subfield $\mathbf{F}_{p}$. Assuming a non-prime field $\mathbf{F}_{q}$ significantly complicates the proofs of Theorem 4 and Lemma 11. So we assume a prime finite field until Sect. 5.
} 
Similarly, by [15, Corollary 2], necessary and sufficient condition for $A$ being $q$ forbidden is

$$
C^{\perp s} \cap \mathbf{F}_{p}^{A}=C_{\max } \cap \mathbf{F}_{p}^{A}=C \cap \mathbf{F}_{p}^{A} .
$$

By a similar argument, we see that Eq. (4) implies Eq. (2).

Next, we give sufficient conditions in terms of the coset distance [11] or the first relative generalized Hamming weight [20]. To do so, we have to slightly modify them. For $(\mathbf{a} \mid \mathbf{b})=\left(a_{1}, \ldots, a_{n} \mid b_{1}, \ldots, b_{n}\right) \in \mathbf{F}_{p}^{n}$, define its symplectic weight $\operatorname{swt}(\mathbf{a} \mid \mathbf{b})=$ $\left|\left\{i:\left(a_{i}, b_{i}\right) \neq(0,0)\right\}\right|$. For $V_{2} \subset V_{1} \subset \mathbf{F}_{p}^{2 n}$, we define their coset distance as $d_{s}\left(V_{1}, V_{2}\right)=\min \left\{\operatorname{swt}(\mathbf{a} \mid \mathbf{b}):(\mathbf{a} \mid \mathbf{b}) \in V_{1} \backslash V_{2}\right\}$.

Theorem 8 If $|A| \leq d_{s}\left(C_{\max }, C\right)-1$, then $A$ is $c$-forbidden. If $|A| \geq n-$ $d_{S}\left(C^{\perp s}, C_{\max }\right)+1$, then $A$ is $c$-qualified.

Example 9 Consider the situation in Example 5. We have $d_{s}\left(C^{\perp}, C_{\max }\right)=1$, which implies that 2 shares form a $c$-qualified set. We also have $d_{s}\left(C_{\max }, C\right)=2$, which implies that 1 share forms a $c$-forbidden set.

Proof (Theorem 8) If $|A| \leq d_{s}\left(C_{\max }, C\right)-1$, then there is no $(\mathbf{a} \mid \mathbf{b}) \in C_{\max } \cap \mathbf{F}_{p}^{A} \backslash C \cap$ $\mathbf{F}_{p}^{A}$ and Eq. (2) holds.

Assume that $|A| \geq n-d_{s}\left(C^{\perp s}, C_{\max }\right)+1$, or equivalently, $|\bar{A}| \leq d_{s}\left(C^{\perp s}, C_{\max }\right)-$ 1. We have $C^{\perp s} \cap \mathbf{F}_{p}^{\bar{A}}=C_{\max } \cap \mathbf{F}_{p}^{\bar{A}}$. We also have $\mathbf{F}_{p}^{\bar{A}}=\operatorname{ker}\left(P_{A}\right)$, which means $\operatorname{dim} P_{A}\left(C^{\perp s}\right)-\operatorname{dim} P_{A}\left(C_{\text {max }}\right)=\operatorname{dim} C^{\perp s}-\operatorname{dim} C_{\max }=k$. Since $\operatorname{dim} C_{\max } \cap \mathbf{F}_{p}^{A}-$ $\operatorname{dim} C \cap \mathbf{F}_{p}^{A}=\operatorname{dim} P_{A}\left(C^{\perp s}\right)-\operatorname{dim} P_{A}\left(C_{\max }\right)=k$, we see that Eq. (1) holds with $A$.

Remark 10 By Remark 7 and a similar argument to the last proof, we see that if $|A| \leq d_{s}\left(C^{\perp s}, C\right)-1$, then $A$ is $q$-forbidden and that if $|A| \geq n-d_{s}\left(C^{\perp s}, C\right)+1$, then $A$ is $q$-qualified. Note that these observations can also be deduced from quantum erasure decoding and [15, Corollary 2] and are not novel.

\section{Amount of information possessed by an intermediate set}

Let $A \subset\{1, \ldots, n\}$ with $A \neq \varnothing$ and $A \neq\{1, \ldots, n\}$. In this section, we study the amount of information possessed by $A$.

Lemma 11 For two classical secrets $\mathbf{m}_{1}$ and $\mathbf{m}_{2}$, we have

$-\operatorname{Tr}_{\bar{A}}\left(\left|f\left(\mathbf{m}_{1}\right) \varphi\right\rangle\left\langle f\left(\mathbf{m}_{\mathbf{1}}\right) \varphi\right|\right)=\operatorname{Tr}_{\bar{A}}\left(\left|f\left(\mathbf{m}_{2}\right) \varphi\right\rangle\left\langle f\left(\mathbf{m}_{\mathbf{2}}\right) \varphi\right|\right)$ if and only if $f\left(\mathbf{m}_{1}\right)$ and $f\left(\mathbf{m}_{2}\right)$ give the same symplectic inner product for all vectors in $C_{\max } \cap \mathbf{F}_{p}^{A}$, and

- $\operatorname{col}\left(\operatorname{Tr}_{\bar{A}}\left(\left|f\left(\mathbf{m}_{1}\right) \varphi\right\rangle\left\langle f\left(\mathbf{m}_{\mathbf{1}}\right) \varphi\right|\right)\right)$ and $\operatorname{col}\left(\operatorname{Tr}_{\bar{A}}\left(\left|f\left(\mathbf{m}_{2}\right) \varphi\right\rangle\left\langle f\left(\mathbf{m}_{\mathbf{2}}\right) \varphi\right|\right)\right)$ are orthogonal to each other if and only if $f\left(\mathbf{m}_{1}\right)$ and $f\left(\mathbf{m}_{2}\right)$ give different symplectic inner products for some vector $(\mathbf{a} \mid \mathbf{b})$ in $C_{\max } \cap \mathbf{F}_{p}^{A}$.

Proof Assume that $f\left(\mathbf{m}_{1}\right)$ and $f\left(\mathbf{m}_{2}\right)$ give the same symplectic inner product for all vectors in $C_{\max } \cap \mathbf{F}_{p}^{A}$. Then, we have $\left\{P_{A}(\mathbf{a} \mid \mathbf{b}):(\mathbf{a} \mid \mathbf{b}) \in f\left(\mathbf{m}_{1}\right)\right\}=\left\{P_{A}(\mathbf{a} \mid \mathbf{b})\right.$ : 
(a|b) $\left.\in f\left(\mathbf{m}_{2}\right)\right\}$, and the encoding procedure on $A$ is the same for $\mathbf{m}_{1}$ and $\mathbf{m}_{2}$, which shows $\operatorname{Tr}_{\bar{A}}\left(\left|f\left(\mathbf{m}_{1}\right) \varphi\right\rangle\left\langle f\left(\mathbf{m}_{\mathbf{1}}\right) \varphi\right|\right)=\operatorname{Tr}_{\bar{A}}\left(\left|f\left(\mathbf{m}_{2}\right) \varphi\right\rangle\left\langle f\left(\mathbf{m}_{\mathbf{2}}\right) \varphi\right|\right)$.

Assume that $f\left(\mathbf{m}_{1}\right)$ and $f\left(\mathbf{m}_{2}\right)$ give different symplectic inner products for some vector (a|b) in $C_{\max } \cap \mathbf{F}_{p}^{A}$. Then, the quantum measurement corresponding to $X(\mathbf{a}) Z$ (b) can be performed only by the participants in $A$ and by [18, Lemma 5] the outcomes for $\left|f\left(\mathbf{m}_{1}\right) \varphi\right\rangle$ and $\left|f\left(\mathbf{m}_{2}\right) \varphi\right\rangle$ are different with probability 1 . This means that $\operatorname{col}\left(\operatorname{Tr}_{\bar{A}}\left(\left|f\left(\mathbf{m}_{1}\right) \varphi\right\rangle\left\langle f\left(\mathbf{m}_{1}\right) \varphi\right|\right)\right)$ and $\operatorname{col}\left(\operatorname{Tr}_{\bar{A}}\left(\left|f\left(\mathbf{m}_{2}\right) \varphi\right\rangle\left\langle f\left(\mathbf{m}_{\mathbf{2}}\right) \varphi\right|\right)\right)$ are orthogonal to each other.

Proposition 12 If $\operatorname{dim} C_{\max } \cap \mathbf{F}_{p}^{A} / C \cap \mathbf{F}_{p}^{A}=\ell$, then the number of density matrices in $\Lambda=\left\{\operatorname{Tr}_{\bar{A}}(|f(\mathbf{m}) \varphi\rangle\langle f(\mathbf{m}) \varphi|): \mathbf{m} \in \mathbf{F}_{p}^{k}\right\}$ is $p^{\ell}$.

For a fixed density matrix $\rho \in \Lambda$, the number of classical secrets $\mathbf{m}$ such that $\rho=\operatorname{Tr}_{\bar{A}}(|f(\mathbf{m}) \varphi\rangle\langle f(\mathbf{m}) \varphi|)$ is exactly $p^{k-\ell}$.

Proof If $P_{A}\left(\mathbf{u}_{1} \mid \mathbf{v}_{1}\right)+P_{A}\left(C_{\max }\right) \neq P_{A}\left(\mathbf{u}_{2} \mid \mathbf{v}_{2}\right)+P_{A}\left(C_{\max }\right)$ for $\left(\mathbf{u}_{i} \mid \mathbf{v}_{i}\right) \in f\left(\mathbf{m}_{i}\right)$ with classical secrets $\mathbf{m}_{i}(i=1,2)$, then by Lemma $11 \operatorname{col}\left(\operatorname{Tr}_{\bar{A}}\left(\left|f\left(\mathbf{m}_{1}\right) \varphi\right\rangle\left\langle f\left(\mathbf{m}_{\mathbf{1}}\right) \varphi\right|\right)\right)$ and $\operatorname{col}\left(\operatorname{Tr}_{\bar{A}}\left(\left|f\left(\mathbf{m}_{2}\right) \varphi\right\rangle\left\langle f\left(\mathbf{m}_{2}\right) \varphi\right|\right)\right)$ are orthogonal. By the assumption, we have $\operatorname{dim} C_{\max } \cap \mathbf{F}_{p}^{A} / C \cap \mathbf{F}_{p}^{A}=\operatorname{dim} P_{A}\left(C^{\perp s}\right) / P_{A}\left(C_{\max }\right)=\ell$. There are $p^{\ell}$ elements in $P_{A}\left(C^{\perp s}\right) / P_{A}\left(C_{\max }\right)$, which shows the first claim.

The composite $\mathbf{F}_{p}$-linear map " $\bmod P_{A}\left(C_{\max }\right)$ " $\circ P_{A} \circ f$ from $\mathbf{F}_{p}^{k}$ to $P_{A}\left(C^{\perp s}\right) /$ $P_{A}\left(C_{\max }\right)$ is surjective. Thus, the dimension of its kernel is $k-\ell$, which shows the second claim.

Definition 13 In light of Proposition 12, the amount of information possessed by a set $A$ of participants is defined as

$$
\left(\log _{2} p\right) \times \operatorname{dim} C_{\max } \cap \mathbf{F}_{p}^{A} / C \cap \mathbf{F}_{p}^{A} .
$$

Remark 14 When the probability distribution of classical secrets $\mathbf{m}$ is uniform, the quantity in Definition 13 is equal to the Holevo information [32, Section 12.1.1] counted in $\log _{2}$. To see this, firstly, the set $\Lambda$ in Proposition 12 consists of nonoverlapping projection matrices and each matrix commutes with every other matrices in $\Lambda$. So the Holevo information is just equal to the classical mutual information [9] between random variable $X$, corresponding to classical secrets in $\mathbf{F}_{p}^{k}$, and random variable $Y$, corresponding to matrices in $\Lambda$, where $Y$ is given as a surjective function of $X$. By Proposition 12, $Y$ has the uniform probability distribution. Therefore, $I(X ; Y)=$ $H(Y)=\log _{2}|\Lambda|=$ Eq. (5).

We say that a secret sharing scheme is $r_{i}$-reconstructible if $|A| \geq r_{i}$ implies $A$ has $i \log _{2} p$ or more bits of information [12]. We say that a secret sharing scheme is $t_{i}$-private if $|A| \leq t_{i}$ implies $A$ has less than $i \log _{2} p$ bits of information [12]. In order to express $r_{i}$ and $t_{i}$ in terms of combinatorial properties of $C$, we introduce a slightly modified version of the relative generalized Hamming weight [20].

Definition 15 For two linear spaces $V_{2} \subset V_{1} \subset \mathbf{F}_{p}^{2 n}$ and $i=1, \ldots, k$, define the $i$ th relative generalized symplectic weight

$$
d_{s}^{i}\left(V_{1}, V_{2}\right)=\min \left\{|A|: \operatorname{dim} \mathbf{F}_{p}^{A} \cap V_{1}-\operatorname{dim} \mathbf{F}_{p}^{A} \cap V_{2} \geq i\right\}
$$


Note that $d_{s}^{1}=d_{s}$. The following theorem generalizes Theorem 8 .

Theorem 16

$$
\begin{aligned}
t_{i} & \geq d_{s}^{i}\left(C_{\max }, C\right)-1, \\
r_{k+1-i} & \leq n-d_{s}^{i}\left(C^{\perp s}, C_{\max }\right)+1 .
\end{aligned}
$$

Example 17 Consider the situation of Example 9. We have $d_{s}^{1}\left(C_{\max }, C\right)=d_{s}^{2}\left(C_{\max }, C\right)$ $=2$, and $d_{s}^{1}\left(C^{\perp s}, C_{\max }\right)=d_{s}^{2}\left(C^{\perp s}, C_{\max }\right)=1$. Unlike the relative generalized Hamming weight, we do not have the strict monotonicity in $i$ of $d_{s}^{i}$.

Proof (Theorem 16) Assume that $|A| \leq t_{i}$. By definition of $d_{s}^{i}, \operatorname{dim} C_{\max } \cap \mathbf{F}_{p}^{A} / C \cap$ $\mathbf{F}_{p}^{A} \leq i-1$, which shows the first claim.

Assume that $|A| \geq r_{i}$. Then, $|\bar{A}| \leq d_{s}^{i}\left(C^{\perp s}, C_{\max }\right)-1$, which implies $\operatorname{dim} C^{\perp s} \cap$ $\mathbf{F}_{p}^{\bar{A}} / C_{\max } \cap \mathbf{F}_{p}^{\bar{A}} \leq i-1$. The last inequality implies $\operatorname{dim} C_{\max } \cap \mathbf{F}_{p}^{A} / C \cap \mathbf{F}_{p}^{A} \geq k-i+1$. which shows the second claim.

\section{Translations to arbitrary finite fields and to the ordinary Hamming weight}

\subsection{Translation to arbitrary finite fields}

Let $q=p^{\mu}$ with $\mu \geq 1$, and $\left\{\gamma_{1}, \ldots, \gamma_{\mu}\right\}$ be a fixed $\mathbf{F}_{p}$-basis of $\mathbf{F}_{q}$. Ashikhmin and Knill [2] proposed the following translation from $\mathbf{F}_{q}$ to $\mathbf{F}_{p}$ for quantum stabilizer codes. Let $M$ be a $\mu \times \mu$ invertible matrix over $\mathbf{F}_{p}$ whose $(i, j)$ element is $\operatorname{Tr}_{q / p}\left(\gamma_{i} \gamma_{j}\right)$, where $\operatorname{Tr}_{q / p}$ is the trace map from $\mathbf{F}_{q}$ to $\mathbf{F}_{p}$. Let $\phi$ be an $\mathbf{F}_{p}$-linear isomorphism sending $\left(a_{1,1}, \ldots, a_{1, \mu}, a_{2,1}, \ldots, a_{n, \mu} \mid b_{1,1}, \ldots, b_{1, \mu}, b_{2,1}, \ldots, b_{n, \mu}\right) \in \mathbf{F}_{p}^{2 \mu n}$ to

$$
\left(\sum_{j=1}^{\mu} a_{1, j} \gamma_{j}, \ldots, \sum_{j=1}^{\mu} a_{n, j} \gamma_{j} \mid \sum_{j=1}^{\mu} b_{1, j}^{\prime} \gamma_{j}, \ldots, \sum_{j=1}^{\mu} b_{n, j}^{\prime} \gamma_{j}\right) \in \mathbf{F}_{q}^{2 n}
$$

where $\left(b_{i, 1}^{\prime}, \ldots, b_{i, \mu}^{\prime}\right)=\left(b_{i, 1}, \ldots, b_{i, \mu}\right) M^{-1}$ for $i=1, \ldots, n$.

Ashikhmin and Knill proved the following.

Proposition 18 [2] Let $C \subset \mathbf{F}_{q}^{2 n}$. Then, $\operatorname{dim}_{\mathbf{F}_{p}} \phi^{-1}(C)=\mu \operatorname{dim}_{\mathbf{F}_{q}} C$, and $\phi^{-1}(C)^{\perp s}=\phi^{-1}\left(C^{\perp s}\right)$, where $\operatorname{dim}_{\mathbf{F}_{q}}$ is the dimension of a linear space considered over $\mathbf{F}_{q}$.

Let $C \subset C_{\text {max }}=C_{\text {max }}^{\perp s} \subset C^{\perp s} \subset \mathbf{F}_{q}^{2 n}$ with $\operatorname{dim}_{\mathbf{F}_{q}} C=n-k$. Then, we have $\phi^{-1}(C) \subset \phi^{-1}\left(C_{\max }\right)=\phi^{-1}\left(C_{\max }\right)^{\perp s} \subset \phi^{-1}(C)^{\perp s} \subset \mathbf{F}_{p}^{2 \mu n}$ and we can construct a secret sharing scheme by $\phi^{-1}(C) \subset \phi^{-1}\left(C_{\max }\right)$. It encodes $k \mu \log _{2} p=k \log _{2} q$ bits of classical secrets $\mathbf{m} \in \mathbf{F}_{q}^{k}$ into $\mu n$ qudits in $\mathbf{C}_{p}$, which can also be seen as $n$ qudits in $\mathbf{C}_{q}$, where $\mathbf{C}_{q}$ is the $q$-dimensional complex linear space. Let $A \subset\{1, \ldots, n\}$. 
By abuse of notation, by $\mathbf{F}_{p}^{A}$ we mean $\left\{\left(a_{1,1}, \ldots, a_{1, \mu}, a_{2,1}, \ldots, a_{n, \mu} \mid b_{1,1}, \ldots, b_{1, \mu}\right.\right.$, $\left.b_{2,1}, \ldots, b_{n, \mu}\right) \in \mathbf{F}_{p}^{2 \mu n}: a_{i, j}=b_{i, j}=0$ for $i \notin A$ and $\left.j=1, \ldots, \mu\right\}$.

We consider each qudit in $\mathbf{C}_{q}$ of the quantum codeword as a share and examine the property of a share set $A$. We have

$$
\operatorname{dim}_{\mathbf{F}_{q}} C_{\max } \cap \mathbf{F}_{q}^{A} / C \cap \mathbf{F}_{q}^{A}=\mu \operatorname{dim}_{\mathbf{F}_{p}} \phi^{-1}\left(C_{\max }\right) \cap \mathbf{F}_{p}^{A} / \phi^{-1}(C) \cap \mathbf{F}_{p}^{A} .
$$

Equation (7) together with Theorem 4 implies

- $A$ is qualified if and only if $\operatorname{dim}_{\mathbf{F}_{q}} C_{\max } \cap \mathbf{F}_{q}^{A} / C \cap \mathbf{F}_{q}^{A}=\operatorname{dim}_{\mathbf{F}_{q}} C_{\max } / C$, and - $A$ is forbidden if and only if $\operatorname{dim}_{\mathbf{F}_{q}} C_{\max } \cap \mathbf{F}_{q}^{A} / C \cap \mathbf{F}_{q}^{A}=0$.

The above observation shows that Theorems 4 and 8 also hold for $\mathbf{F}_{q}$. In addition, Eq. (7) means that a share set $A$ has $\left(\log _{2} q \times \operatorname{dim}_{\mathbf{F}_{q}} C_{\max } \cap \mathbf{F}_{q}^{A} / C \cap \mathbf{F}_{q}^{A}\right)$-bits of information about the secret $\mathbf{m} \in \mathbf{F}_{q}^{k}$, also generalizes the proof argument of Theorem 16, and implies that Theorem 16 also holds for $\mathbf{F}_{q}$. In the sequel, we consider a qudit in $\mathbf{C}_{q}$ as each share and dim means the dimension over $\mathbf{F}_{q}$.

\subsection{Translation to the Hamming distance and the hermitian inner product}

Many of results in the symplectic construction of quantum error-correcting codes over $\mathbf{F}_{q}$ are translated to $\mathbf{F}_{q^{2}}$-linear codes with the hermitian inner product [2,18,29]. For $\mathbf{x} \in \mathbf{F}_{q^{2}}^{n}$ define $\mathbf{x}^{q}$ as the component-wise $q$ th power of $\mathbf{x}$. For two vectors $\mathbf{x}, \mathbf{y} \in \mathbf{F}_{q^{2}}$, define the hermitian inner product as $\langle\mathbf{x}, \mathbf{y}\rangle_{h}=\left\langle\mathbf{x}^{q}, \mathbf{y}\right\rangle_{E}$. For $D \subset \mathbf{F}_{q^{2}}^{n}, D^{\perp h}$ denotes the orthogonal space of $D$ with respect to the hermitian inner product.

Only in Sects. 5.2, 5.3 and 5.4, for $A \subset\{1, \ldots, n\}$, define $\mathbf{F}_{q}^{A}=\left\{\left(a_{1}, \ldots, a_{n}\right) \in\right.$ $\mathbf{F}_{q}^{n}: a_{i}=0$ for $\left.i \notin A\right\}$, and define $P_{A}$ to be the projection map onto $A$, that is, $P_{A}\left(a_{1}, \ldots, a_{n}\right)=\left(a_{i}\right)_{i \in A}$.

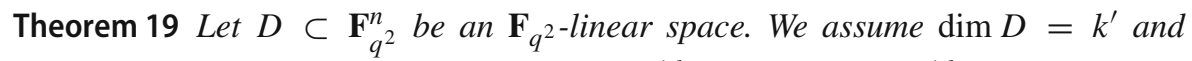
there exists $D_{\max }$ such that $D \subset D_{\max } \subset D^{\perp h}$ and $D_{\max }=D_{\max }^{\perp h}$, which implies $\operatorname{dim} D_{\max }=n / 2$. Then, $D$ defines a secret sharing scheme based on the quantum stabilizer defined by $D$ encoding $n-2 k^{\prime}$ symbols in $\mathbf{F}_{q}$. A set $A \subset\{1, \ldots, n\}$ is c-qualified if and only if $\operatorname{dim} D_{\max } / D=\operatorname{dim} D_{\max } \cap \mathbf{F}_{q^{2}}^{A} / D \cap \mathbf{F}_{q^{2}}^{A}$. A set $A \subset$ $\{1, \ldots, n\}$ is c-forbidden if and only if $0=\operatorname{dim} D_{\max } \cap \mathbf{F}_{q^{2}}^{A} / D \cap \mathbf{F}_{q^{2}}^{A}$. If $|A| \geq$ $n-d_{H}\left(D^{\perp h}, D_{\max }\right)+1$, then $A$ is $c$-qualified, and if $|A| \leq d_{H}\left(D_{\max }, D\right)-1$, then A is c-forbidden, where $d_{H}$ is the coset distance [11], or equivalently, the first relative generalized Hamming weight [20].

Proof The proof is almost same as [18].

Example 20 Consider the situation in Example 9. Then, $D=\{0\}$ and $D_{\max }$ is the one-dimensional $\mathbf{F}_{4}$-linear space spanned by $(1,1)$. 


\subsection{Translation to the Hamming distance and the Euclidean inner product}

Let $C_{2} \subset C_{1} \subset \mathbf{F}_{q}^{n}$. A method to construct symplectic-self-orthogonal $C \subset \mathbf{F}_{q}^{2 n}$ is to use $\left\{(\mathbf{a} \mid \mathbf{b}): \mathbf{a} \in C_{2}, \mathbf{b} \in C_{1}^{\perp E}\right\}$ as $C[5,18]$, where " $\perp E$ " denotes the Euclidean dual. We have $C^{\perp s}=\left\{(\mathbf{a} \mid \mathbf{b}): \mathbf{a} \in C_{1}, \mathbf{b} \in C_{2}^{\perp E}\right\}$.

Example 21 Example 6 can also be described by $C_{2}=\{0\}, C_{1}=\mathbf{F}_{2}^{2}$, and $C_{\max }^{\prime}=$ $\left\{(\mathbf{a} \mid \mathbf{b}): \mathbf{a} \in C_{2}, \mathbf{b} \in C_{2}^{\perp E}\right\}$.

Remark 22 A suitable choice of $C_{\max }$ is unclear as of this writing. A valid choice is $C_{\max }^{\prime}=\left\{(\mathbf{a} \mid \mathbf{b}): \mathbf{a} \in C_{2}, \mathbf{b} \in C_{2}^{\perp E}\right\}$, which gives the standard encoding [6,38] of the CSS codes. But this choice gives no advantage over the purely classical secret sharing constructed from linear codes $C_{2} \subset C_{1}$ [3,7,19,23]. Because the necessary and sufficient condition for $c$-qualified $A$ is $\operatorname{dim} P_{A}\left(C_{1}\right) / P_{A}\left(C_{2}\right)=\operatorname{dim} C_{1} / C_{2}$ and the necessary and sufficient condition for $c$-forbidden $A$ is $\operatorname{dim} P_{A}\left(C_{1}\right) / P_{A}\left(C_{2}\right)=0$ by combining [26, Section 4.1] and [33, Theorem 1], which are exactly same [12] as those of the purely classical secret sharing constructed from $C_{2} \subset C_{1}$.

Theorem 23 Let $E \subset \mathbf{F}_{q}^{n}$ be the $\mathbf{F}_{q}$-linear space. We assume $\operatorname{dim} E=k^{\prime}$, and there exists $E_{\max }$ such that $E \subset E_{\max } \subset E^{\perp E}$ and $E_{\max }=E_{\max }^{\perp E}$, which implies $\operatorname{dim} E_{\max }=n / 2$. Then, $E$ defines a secret sharing scheme based on the quantum stabilizer defined by $E$ encoding $n-2 k^{\prime}$ symbols in $\mathbf{F}_{q}$. A set $A \subset\{1, \ldots, n\}$ is $c$ qualified if and only if $\operatorname{dim} E_{\max } / E=\operatorname{dim} E_{\max } \cap \mathbf{F}_{q}^{A} / E \cap \mathbf{F}_{q}^{A}$. A set $A \subset\{1, \ldots, n\}$ is $c$-forbidden if and only if $0=\operatorname{dim} E_{\max } \cap \mathbf{F}_{q}^{A} / E \cap \mathbf{F}_{q}^{A}$. If $|A| \geq n-d_{H}\left(E^{\perp E}, E_{\max }\right)+1$, then $A$ is $c$-qualified, and if $|A| \leq d_{H}\left(E_{\max }, E\right)-1$, then $A$ is $c$-forbidden.

Proof The proof is almost same as [18].

Example 24 Example 3 is restored by choosing $E=\{0\}, E^{\perp E}=\mathbf{F}_{2}^{2}$, and $E_{\max }$ as the $\mathbf{F}_{2}$-linear space spanned by $(1,1)$. Thus, we see that Theorem 23, in contrast to Remark 22 , can provide a secret sharing scheme with an advantage over purely classical secret sharing.

\subsection{Construction by the Reed-Solomon codes}

Also as an example of Theorem 23, an elementary construction by the Reed-Solomon (RS) codes will be shown below. In Sect. 5.4, assume that $n=q$ and $k$ are positive even integers. Fix $n$ distinct elements $\alpha_{1}, \ldots, \alpha_{n} \in \mathbf{F}_{q}$. Define

$$
\operatorname{RS}(n, k)=\left\{\left(g\left(\alpha_{1}\right), \ldots, g\left(\alpha_{n}\right)\right): g(x) \in \mathbf{F}_{q}[x], \operatorname{deg} g(x)<k\right\} .
$$

Then, $\operatorname{RS}(n, k)^{\perp E}=\operatorname{RS}(n, n-k)$ as $n=q$.

For a linear space $V \subset \mathbf{F}_{q}^{n}$, its $j$ th generalized Hamming weight $d_{H}^{j}(V)$ is defined by [34]

$$
d_{H}^{j}(V)=\min \left\{|A|: \operatorname{dim} \mathbf{F}_{q}^{A} \cap V \geq j\right\}
$$


For RS codes, $d_{H}^{j}(\mathrm{RS}(n, k))=n-k+j[34]$.

Define

$$
\begin{aligned}
C & =\{(\mathbf{a} \mid \mathbf{b}): \mathbf{a}, \mathbf{b} \in \operatorname{RS}(n,(n-k) / 2)\} \subset \mathbf{F}_{q}^{2 n}, \\
C_{\max } & =\{(\mathbf{a} \mid \mathbf{b}): \mathbf{a}, \mathbf{b} \in \operatorname{RS}(n, n / 2)\} \subset \mathbf{F}_{q}^{2 n},
\end{aligned}
$$

which correspond to $E=\operatorname{RS}(n,(n-k) / 2)$ and $E_{\max }=\operatorname{RS}(n, n / 2)$ in Theorem 23 . Then, we have

$$
\begin{aligned}
C_{\max } & =C_{\max }^{\perp s}, \\
C^{\perp s} & =\{(\mathbf{a} \mid \mathbf{b}): \mathbf{a}, \mathbf{b} \in \operatorname{RS}(n,(n+k) / 2)\}, \\
\operatorname{dim} C & =n-k, \\
d_{s}^{j}\left(C_{\max }, C\right) & \geq d_{s}^{j}\left(C_{\max },\{\mathbf{0}\}\right) \geq d_{H}^{\lceil j / 2\rceil}(\operatorname{RS}(n, n / 2))=\left\lceil\frac{n+j}{2}\right\rceil, \\
d_{s}^{j}\left(C^{\perp s}, C_{\max }\right) & \geq d_{s}^{j}\left(C^{\perp s},\{\mathbf{0}\}\right) \geq d_{H}^{\lceil j / 2\rceil}(\operatorname{RS}(n,(n+k) / 2))=\left\lceil\frac{n-k+j}{2}\right\rceil .
\end{aligned}
$$

In particular, we have $d_{s}\left(C_{\max }, C\right) \geq n / 2+1$ and $d_{s}\left(C^{\perp s}, C_{\max }\right) \geq(n-k) / 2+1$. For a set of participants $A \subseteq\{1, \ldots, n\}, A$ is forbidden if $|A| \leq n / 2$ and $A$ is qualified if $|A| \geq(n+k) / 2$, by Theorem 8 or also by Theorem 23 .

By Theorem 16, $t_{i} \geq\lceil(n+i) / 2\rceil-1$ and $r_{i} \leq\lceil(n+i) / 2\rceil$. In addition, by the definitions of $r_{i}$ and $t_{i}$, we have $r_{i} \geq t_{i}+1$. So we see that $t_{i}=\lceil(n+i) / 2\rceil-1$ and $r_{i}=\lceil(n+i) / 2\rceil$, which also imply that inequalities (8) and (9) are in fact equalities by Theorem 16. Let $\rho_{A}$ be the density matrix of quantum shares in a share set $A \subseteq$ $\{1, \ldots, n\}$. Until the end of Sect. 5.4, assume that classical secrets $\mathbf{m}$ are uniformly distributed. From Remark 14 and those exact values of $r_{i}$ and $t_{i}$ for $i=1, \ldots, k$, we see that the Holevo information [32, Section 12.1.1] (quantum counterpart of the mutual information [9]) between $\mathbf{m}$ and $\rho_{A}$ is

$$
I\left(\mathbf{m} ; \rho_{A}\right)=\left\{\begin{array}{cl}
0 & \text { if } 0 \leq|A| \leq \frac{n}{2} \\
2\left(|A|-\frac{n}{2}\right) \log _{2} q & \text { if } \frac{n}{2} \leq|A| \leq \frac{n+k}{2} \\
k \log _{2} q & \text { if } \frac{n+k}{2} \leq|A| \leq n .
\end{array}\right.
$$

Observe here that one increment of the share size increases the Holevo information by two $\mathbf{F}_{q}$ symbols. This is in sharp contrast with the classical linear secret sharing [3, $7,19,23$ ], because one increment of the share size can increase the mutual information by at most one $\mathbf{F}_{q}$ symbol in classical linear secret sharing, when each share is one $\mathbf{F}_{q}$ symbol. Observe also that we have completely determined the classical access structure of this quantum secret sharing scheme.

\section{Gilbert-Varshamov-type existential condition}

In this section, we give a sufficient condition for existence of $C \subset C_{\max }=C_{\max }^{\perp s} \subset$ $C^{\perp s} \subset \mathbf{F}_{q}^{2 n}$, with given parameters. 
Theorem 25 If positive integers $n, k, \delta_{t}, \delta_{r}$ satisfy

$$
\frac{q^{n+k}-q^{n}}{q^{2 n}-1} \sum_{i=1}^{\delta_{r}-1}\left(\begin{array}{l}
n \\
i
\end{array}\right)\left(q^{2}-1\right)^{i}+\frac{q^{n}-q^{n-k}}{q^{2 n}-1} \sum_{i=1}^{\delta_{t}-1}\left(\begin{array}{l}
n \\
i
\end{array}\right)\left(q^{2}-1\right)^{i}<1
$$

then there exist $C \subset C_{\max }=C_{\max }^{\perp s} \subset C^{\perp s} \subset \mathbf{F}_{q}^{2 n}$ such that $\operatorname{dim} C=n-k$, $d_{s}\left(C^{\perp s}, C_{\max }\right) \geq \delta_{r}$ and $d_{s}\left(C_{\max }, C\right) \geq \delta_{t}$.

Proof The following argument is similar to the proof of Gilbert-Varshamov bound for stabilizer codes [4]. Let $\operatorname{Sp}(q, n)$ be the set of invertible matrices on $\mathbf{F}_{q}^{2 n}$ that does not change the values of the symplectic inner product. Let $A(k)$ be the set of pairs of linear spaces $(V, W)$ such that $\operatorname{dim} V=n-k$ and $V \subset W=W^{\perp s} \subset V^{\perp s} \subset \mathbf{F}_{q}^{2 n}$. For e $\in \mathbf{F}_{q}^{2 n}$, define $B_{V}(k, \mathbf{e})=\left\{(V, W) \in A(k): \mathbf{e} \in V^{\perp s} \backslash W\right\}$ and $B_{W}(k, \mathbf{e})=$ $\{(V, W) \in A(k): \mathbf{e} \in W \backslash V\}$. It is known in mathematics [1, Chapter 7]

- for nonzero $\mathbf{e}_{1}, \mathbf{e}_{2} \in \mathbf{F}_{q}^{2 n}$, there exists $M \in \operatorname{Sp}(q, n)$ such that $M \mathbf{e}_{1}=\mathbf{e}_{2}$, and

- for $\left(V_{1}, W_{1}\right),\left(V_{2}, W_{2}\right) \in A(k)$, there exists $M \in \operatorname{Sp}(q, n)$ such that $M V_{1}=V_{2}$ and $M W_{1}=W_{2}$.

For nonzero $\mathbf{e}_{1}, \mathbf{e}_{2} \in \mathbf{F}_{q}^{2 n}$ with $M_{1} \mathbf{e}_{1}=\mathbf{e}_{2}\left(M_{1} \in \operatorname{Sp}(q, n)\right)$ and some fixed $\left(V_{1}, W_{1}\right) \in A(k)$, we have

$$
\begin{aligned}
\mid & B_{W}\left(k, \mathbf{e}_{1}\right) \mid \\
& =\left|\left\{(V, W) \in A(k): \mathbf{e}_{1} \in W \backslash V\right\}\right| \\
& =\left|\left\{\left(M V_{1}, M W_{1}\right): \mathbf{e}_{1} \in M W \backslash M V, M \in \operatorname{Sp}(q, n)\right\}\right| \\
& =\left|\left\{\left(M_{1}^{-1} M V_{1}, M_{1}^{-1} M W_{1}\right): \mathbf{e}_{1} \in M_{1}^{-1} M W \backslash M_{1}^{-1} M V, M \in \operatorname{Sp}(q, n)\right\}\right| \\
& =\left|\left\{\left(M V_{1}, M W_{1}\right): M_{1} \mathbf{e}_{1} \in M W \backslash M V, M \in \operatorname{Sp}(q, n)\right\}\right| \\
& =\left|\left\{\left(M V_{1}, M W_{1}\right): \mathbf{e}_{2} \in M W \backslash M V, M \in \operatorname{Sp}(q, n)\right\}\right| \\
& =\left|\left\{(V, W) \in A(k): \mathbf{e}_{2} \in W \backslash V\right\}\right| \\
& =\left|B_{W}\left(k, \mathbf{e}_{2}\right)\right| .
\end{aligned}
$$

By a similar argument, we also see $\left|B_{V}\left(k, \mathbf{e}_{1}\right)\right|=\left|B_{V}\left(k, \mathbf{e}_{2}\right)\right|$.

For each $(V, W) \in A(k)$, the number of e such that $\mathbf{e} \in W \backslash V$ is $|W|-|V|=$ $q^{n}-q^{n-k}$. The number of triples $(\mathbf{e}, V, W)$ such that $\mathbf{0} \neq \mathbf{e} \in W \backslash V$ is

$$
\sum_{\mathbf{0} \neq \mathbf{e} \in \mathbf{F}_{q}^{2 n}}\left|B_{W}(k, \mathbf{e})\right|=|A(k)| \times\left(q^{n}-q^{n-k}\right),
$$

which implies

$$
\frac{\left|B_{W}(k, \mathbf{e})\right|}{|A(k)|}=\frac{q^{n}-q^{n-k}}{q^{2 n}-1}
$$

Similarly, we have

$$
\frac{\left|B_{V}(k, \mathbf{e})\right|}{|A(k)|}=\frac{q^{n+k}-q^{n}}{q^{2 n}-1} .
$$


If there exists $(V, W) \in A(k)$ such that $(V, W) \notin B_{V}\left(k, \mathbf{e}_{1}\right)$ and $(V, W) \notin B_{V}\left(k, \mathbf{e}_{2}\right)$ for all $1 \leq \operatorname{swt}\left(\mathbf{e}_{1}\right) \leq \delta_{r}-1$ and $1 \leq \operatorname{swt}\left(\mathbf{e}_{2}\right) \leq \delta_{t}-1$, then there exists a pair of $(V, W)$ with the desired properties. The number of e such that $1 \leq \operatorname{swt}(\mathbf{e}) \leq \delta-1$ is given by

$$
\sum_{i=1}^{\delta-1}\left(\begin{array}{l}
n \\
i
\end{array}\right)\left(q^{2}-1\right)^{i} .
$$

By combining Eqs. (11), (12) and (13), we see that Eq. (10) is a sufficient condition for ensuring the existence of $(V, W)$ required in Theorem 25 .

We will derive an asymptotic form of Theorem 25 .

Theorem 26 Let $R, \epsilon_{t}$ and $\epsilon_{r}$ be nonnegative real numbers $\leq 1$. Define $h_{q}(x)=$ $-x \log _{q} x-(1-x) \log _{q}(1-x)$. For sufficiently large $n$, if

$$
\begin{aligned}
& h_{q}\left(\epsilon_{t}\right)+\epsilon_{t} \log _{q}\left(q^{2}-1\right)<1 \text { and } \\
& h_{q}\left(\epsilon_{r}\right)+\epsilon_{r} \log _{q}\left(q^{2}-1\right)<1-R,
\end{aligned}
$$

then there exist $C \subset C_{\max } \subset C^{\perp s} \subset \mathbf{F}_{q}^{2 n}$ such that $\operatorname{dim} C=n-\lfloor n R\rfloor$, $d_{s}\left(C^{\perp s}, C_{\max }\right) \geq\left\lfloor n \epsilon_{r}\right\rfloor$ and $d_{s}\left(C_{\max }, C\right) \geq\left\lfloor n \epsilon_{t}\right\rfloor$.

Proof Proof can be done by almost the same argument as [30, Section III.C].

Theorem 26 has a striking implication that we can construct a secret sharing scheme with roughly $19 \%$ of participants being forbidden independently of the size (i.e., $R$ in Theorem 26) of classical secrets for $q=2$ and large $n$, as $h_{2}(0.19)+0.19 \log _{2} 3 \simeq 1$. Such properties cannot be realized by classical shares.

\section{Conclusion}

In this paper, we considered construction of secret sharing schemes for classical secrets by quantum stabilizer codes and clarified their access structures, that is, qualified and forbidden sets, in terms of underlying quantum stabilizers. We expressed our findings in terms of linear spaces over finite fields associated with the quantum stabilizers and gave sufficient conditions for qualified and forbidden sets in terms of combinatorial parameters of the linear spaces over finite fields. It allowed us to use classical coding theoretic techniques, such as the Gilbert-Varshamov-type argument, and we obtained a sufficient condition for existence of a secret sharing scheme with given parameters. By using that sufficient condition, we demonstrated that there exist infinitely many quantum stabilizers with which associated access structures cannot be realized by any purely classical information processing. We have not thoroughly considered code construction, which is a future research agenda.

Acknowledgements The research problem was formulated in a discussion with Diego Ruano during the author's stay at the University of Valladolid, Spain. Without the discussion with him, this paper would not exist. The author also thanks an anonymous reviewer for pointing out a related paper [13]. 
Open Access This article is distributed under the terms of the Creative Commons Attribution 4.0 International License (http://creativecommons.org/licenses/by/4.0/), which permits unrestricted use, distribution, and reproduction in any medium, provided you give appropriate credit to the original author(s) and the source, provide a link to the Creative Commons license, and indicate if changes were made.

\section{References}

1. Aschbacher, M.: Finite Group Theory, Cambridge Studies in Advanced Mathematics, vol. 10, 2nd edn. Cambridge University Press, Cambridge (2000). https://doi.org/10.1017/CBO9781139175319

2. Ashikhmin, A., Knill, E.: Nonbinary quantum stabilizer codes. IEEE Trans. Inf. Theory 47(7), 30653072 (2001). https://doi.org/10.1109/18.959288

3. Bains, T.: Generalized Hamming weights and their applications to secret sharing schemes. Master's thesis, University of Amsterdam (2008). https://esc.fnwi.uva.nl/thesis/apart/math/thesis.php?start=391 (supervised by R. Cramer, G. van der Geer, and R. de Haan)

4. Calderbank, A.R., Rains, E.M., Shor, P.W., Sloane, N.J.A.: Quantum error correction and orthogonal geometry. Phys. Rev. Lett. 78(3), 405-408 (1997). https://doi.org/10.1103/PhysRevLett.78.405

5. Calderbank, A.R., Rains, E.M., Shor, P.W., Sloane, N.J.A.: Quantum error correction via codes over GF(4). IEEE Trans. Inf. Theory 44(4), 1369-1387 (1998). https://doi.org/10.1109/18.681315

6. Calderbank, A.R., Shor, P.W.: Good quantum error-correcting codes exist. Phys. Rev. A 54(2), 10981105 (1996). https://doi.org/10.1103/PhysRevA.54.1098

7. Chen, H., Cramer, R., Goldwasser, S., de Haan, R., Vaikuntanathan, V.: Secure computation from random error correcting codes. In: Advances in Cryptology-EUROCRYPT 2007, Lecture Notes in Computer Science, vol. 4515, pp. 291-310. Springer (2007). https://doi.org/10.1007/978-3-54072540-4_17

8. Cleve, R., Gottesman, D., Lo, H.K.: How to share a quantum secret. Phys. Rev. Lett. 83(3), 648-651 (1999). https://doi.org/10.1103/PhysRevLett.83.648

9. Cover, T.M., Thomas, J.A.: Elements of Information Theory, 2nd edn. Wiley Interscience, New York (2006). https://doi.org/10.1002/047174882X

10. dela Cruz, R., Meyer, A., Solé, P.: Extension of Massey scheme for secret sharing. In: Proceedings of ITW 2010. Dublin, Ireland (2010). https://doi.org/10.1109/CIG.2010.5592719

11. Duursma, I.M., Park, S.: Coset bounds for algebraic geometric codes. Finite Fields Appl. 16(1), 36-55 (2010). https://doi.org/10.1016/j.ffa.2009.11.006

12. Geil, O., Martin, S., Matsumoto, R., Ruano, D., Luo, Y.: Relative generalized Hamming weights of one-point algebraic geometric codes. IEEE Trans. Inf. Theory 60(10), 5938-5949 (2014). https://doi. org/10.1109/TIT.2014.2345375

13. Gheorghiu, V.: Generalized semiquantum secret-sharing schemes. Phys. Rev. A 85(5), 052309 (2012). https://doi.org/10.1103/PhysRevA.85.052309

14. Gottesman, D.: Class of quantum error-correcting codes saturating the quantum Hamming bound. Phys. Rev. A 54(3), 1862-1868 (1996). https://doi.org/10.1103/PhysRevA.54.1862

15. Gottesman, D.: Theory of quantum secret sharing. Phys. Rev. A 61(4), 042311 (2000). https://doi.org/ 10.1103/PhysRevA.61.042311

16. Hillery, M., Bužek, V., Berthiaume, A.: Quantum secret sharing. Phys. Rev. A 59, 1829-1834 (1999). https://doi.org/10.1103/PhysRevA.59.1829

17. Karlsson, A., Koashi, M., Imoto, N.: Quantum entanglement for secret sharing and secret splitting. Phys. Rev. A 59, 162-168 (1999). https://doi.org/10.1103/PhysRevA.59.162

18. Ketkar, A., Klappenecker, A., Kumar, S., Sarvepalli, P.K.: Nonbinary stabilizer codes over finite fields. IEEE Trans. Inf. Theory 52(11), 4892-4924 (2006). https://doi.org/10.1109/TIT.2006.883612

19. Kurihara, J., Uyematsu, T., Matsumoto, R.: Secret sharing schemes based on linear codes can be precisely characterized by the relative generalized Hamming weight. IEICE Trans. Fundam. E95A(11), 2067-2075 (2012). https://doi.org/10.1587/transfun.E95.A.2067

20. Luo, Y., Mitrpant, C., Han Vinck, A.J., Chen, K.: Some new characters on the wire-tap channel of type II. IEEE Trans. Inf. Theory 51(3), 1222-1229 (2005). https://doi.org/10.1109/TIT.2004.842763

21. Marin, A., Markham, D.: Equivalence between sharing quantum and classical secrets and error correction. Phys. Rev. A 88(4), 042332 (2013). https://doi.org/10.1103/PhysRevA.88.042332 
22. Markham, D., Sanders, B.C.: Graph states for quantum secret sharing. Phys. Rev. A 78(4), 042309 (2008). https://doi.org/10.1103/PhysRevA.78.042309

23. Martínez-Peñas, U.: On the similarities between generalized rank and Hamming weights and their applications to network coding. IEEE Trans. Inf. Theory 62(7), 4081-4095 (2016). https://doi.org/10. 1109/TIT.2016.2570238

24. Matsumoto, R.: Quantum stabilizer codes can realize access structures impossible by classical secret sharing. IEICE Trans. Fundam. E100-A(12), 2738-2739 (2017). https://doi.org/10.1587/transfun. E100.A.2738

25. Matsumoto, R.: Unitary reconstruction of secret for stabilizer based quantum secret sharing. Quant. Inf. Process. 16(8), 202 (2017). https://doi.org/10.1007/s11128-017-1656-1

26. Matsumoto, R.: Coding theoretic construction of quantum ramp secret sharing. IEICE Trans. Fundam. E101-A(8), 1215-1222 (2018). https://doi.org/10.1587/transfun.E101.A.1215

27. Matsumoto, R.: Exploring quantum supremacy in access structures of secret sharing by coding theory. In: Proceedings of 2018 ISITA, pp. 331-333. Singapore (2018). https://doi.org/10.23919/ISITA.2018. 8664271

28. Matsumoto, R.: Classical access structures of ramp secret sharing based on quantum stabilizer codes. In: Proceedings of WCC 2019, paper no. 2. Saint-Jacut-de-la-Mer, France (2019). https://www.lebesgue. $\mathrm{fr} /$ sites/default/files/proceedings_WCC/WCC_2019_paper_2.pdf

29. Matsumoto, R., Uyematsu, T.: Constructing quantum error-correcting codes for $p^{m}$-state systems from classical error-correcting codes. IEICE Trans. Fundam. E83-A(10), 1878-1883 (2000)

30. Matsumoto, R., Uyematsu, T.: Lower bound for the quantum capacity of a discrete memoryless quantum channel. J. Math. Phys. 43(9), 4391-4403 (2002). https://doi.org/10.1063/1.1497999

31. McEliece, R.J., Sarwate, D.V.: On sharing secrets and Reed-Solomon codes. Commun. ACM 24(9), 583-584 (1981). https://doi.org/10.1145/358746.358762

32. Nielsen, M.A., Chuang, I.L.: Quantum Computation and Quantum Information. Cambridge University Press, Cambridge (2000). https://doi.org/10.1017/CBO9780511976667

33. Ogawa, T., Sasaki, A., Iwamoto, M., Yamamoto, H.: Quantum secret sharing schemes and reversibility of quantum operations. Phys. Rev. A 72(3), 032318 (2005). https://doi.org/10.1103/PhysRevA.72. 032318

34. Pless, V.S., Huffman, W.C., Brualdi, R.A.: An introduction to algebraic codes. In: Pless, V.S., Huffman, W.C. (eds.) Handbook of Coding Theory, pp. 3-139. Elsevier, Amsterdam (1998)

35. Sarvepalli, P.K.: Nonthreshold quantum secret-sharing schemes in the graph-state formalism. Phys. Rev. A 86(4), 042303 (2012). https://doi.org/10.1103/PhysRevA.86.042303

36. Shamir, A.: How to share a secret. Commun. ACM 22(11), 612-613 (1979). https://doi.org/10.1145/ 359168.359176

37. Smith, A.D.: Quantum secret sharing for general access structures (2000). arXiv:quant-ph/0001087

38. Steane, A.M.: Multiple particle interference and quantum error correction. Proc. R. Soc. Lond. Ser. A 452(1954), 2551-2577 (1996). https://doi.org/10.1098/rspa.1996.0136

39. Stinson, D.R.: Cryptography Theory and Practice, 3rd edn. Chapman \& Hall, London (2006). https:// doi.org/10.1201/9781420057133

Publisher's Note Springer Nature remains neutral with regard to jurisdictional claims in published maps and institutional affiliations. 